\title{
Algoritmo de Aprendizaje-Q aplicado al Control de la Calidad de Potencia en Redes de Media Tensión con Generación Distribuida
}

\author{
Miguel Piumetto(1), Juan C. Gómez ${ }^{(2)}$, Julián A. Pucheta(1), Jorge C. Vaschetti( ${ }^{(3)}$ \\ (1) Facultad de Ciencias Exactas Físicas y Naturales, Univ. Nacional de Córdoba, Av. Vélez Sarsfield 1611, \\ Ciudad Universitaria, X5000JJC Córdoba-Argentina. (mpiumetto@efn.uncor.edu; jpucheta@efn.uncor.edu) \\ (2) Facultad de Ingeniería, Universidad Nacional de Río Cuarto, Ruta Nacional 36 Km. 601, X5804BYA Río \\ Cuarto, Córdoba - Argentina. (jcgomez@ing.unrc.edu.ar) \\ (3) Facultad Regional Córdoba, Universidad Tecnológica Nacional, Maestro M. López esq. Cruz Roja \\ Argentina, Ciudad Universitaria, X5016ZAA Córdoba, Argentina. (jvaschetti@hotmail.com)
}

Recibido Oct. 22, 2014; Aceptado Dic. 23, 2014; Versión final recibida Feb. 1, 2015

\begin{abstract}
Resumen
En este trabajo se estudia la implementación de programación dinámica como lógica de control de la calidad de potencia en sistemas de distribución eléctrica de media tensión con generación distribuida. Esto con el fin de optimizar los indicadores de calidad de potencia en conjunto con los costos de operación y para mejorar la eficiencia energética, como también aprovechar la capacidad de transporte y de distribución de la red. Con este propósito se trabajó sobre un sistema real de distribución de la empresa EPEC de la Provincia de Córdoba, Argentina, modelando el comportamiento real del mismo mediante el software ATP/EMTP. Se construye luego una plataforma para el control usando MATLAB y se desarrolló un algoritmo para procesar la información y ajustar el sistema bajo las nuevas condiciones de funcionamiento. Los resultados muestran la posibilidad de controlar, regular y mejorar los indicadores de calidad, optimizando el ingreso de la generación distribuida.
\end{abstract}

Palabras clave: programación dinámica, redes de media tensión, calidad de potencia, generación distribuida, optimización numérica

\section{Q-Learning Algorithm applied to Control of Power Quality in Medium Voltage Networks with Distributed Generation}

\begin{abstract}
In this work the implementation of dynamic programming is studied as control logic of the power quality in medium voltage electric distribution systems with distributed generation. This was done with the aim of optimizing the power quality indicators and the operation costs; and also to improve the energy efficiency, as well as to take advantage of the transport capacity and of the distribution of the network. With this purpose the work was carried out on a real distribution system belonging to the EPEC company of the Córdoba province in Argentina. The real system behavior was modeled by means of the software ATP/EMTP. Afterwards, a platform was built for the control using MATLAB and an algorithm was developed to process the information and to adjust the system under the new operation conditions. The results indicate that it is possible to control, to regulate and to improve quality indicators, optimizing the distributed generation entrance.
\end{abstract}

Keywords: dynamic programming, medium voltage grids, power quality, distributed generation, numerical optimization 


\section{INTRODUCCIÓN}

En el sistema actual, la energía eléctrica llega a los consumidores principalmente por medio de la Empresa Distribuidora (ED), la cual adquiere la energía del sistema central de generación, cumpliendo ésta sólo el rol de distribución de energía eléctrica. En un escenario competitivo, pueden ingresar al mercado eléctrico nuevos agentes, los cuales son independientes de la ED, ofertando inyección de energía en el sistema de distribución por medio de unidades de Generación Distribuida (GD), pasando a ser los clientes entonces, usuarios pasivos/activos.

El objetivo primordial de las ED de energía eléctrica, es la entrega de energía de manera oportuna y cumpliendo con las características técnicas definidas por los entes reguladores. Buscando garantizar que el sistema de distribución cumple adecuadamente con su objetivo, las ED desarrollan diferentes procesos de planificación del sistema. Estos procesos son abordados desde diferentes ópticas o enfoques: algunos centrados en cumplir las características técnicas, otros en determinar las condiciones de servicio adecuadas o solamente teniendo en cuenta las pérdidas técnicas y costos asociados, pero siempre, considerando las condiciones ambientales y de entorno, que afectan la operación y desempeño del sistema pero en última instancia, pretendiendo cumplir con la entrega de un producto bajo las condiciones establecidas por la regulación y buscando los mejores rendimientos económicos para la ED (Bernal, 1998).

Hoy en día la calidad del producto eléctrico en los Sistemas de Distribución (SD) de Media Tensión (MT) está siendo fiscalizada de manera más rigurosa debido a las exigencias de los usuarios. Una falla en el SD modifica severamente los índices de calidad de la ED, generando con ello importantes pérdidas económicas. Encontrar la red de MT que opere en forma óptima con los elementos más confiables, hará que disminuya su probabilidad de falla, lo que implica mejores índices de calidad para la ED. Es importante notar que la complejidad asociada a la búsqueda de ese estado no es menor, puesto que normalmente una ED conecta cientos de cargas, además, el problema presenta restricciones técnicas en su operación, ya que los clientes deben permanecer conectados, y aparece el ingreso de nuevas fuentes de energía, buscando mantener siempre los indicadores de calidad de producto y de servicio técnico, establecidos por la legislación vigente.

El problema de optimización de la redes ha sido abordado utilizando diversos métodos y funcionales, observando trabajos de acuerdo al funcional, empleando criterios como minimización de pérdidas técnicas, funcionales relacionados con confiabilidad o tiempos de restauración y funcionales de costos de operación. Estudios en la literatura internacional muestran dichas tendencias, como (McDermott et al., 1999) que utiliza un funcional de costo que minimiza pérdidas eléctricas, mientras que en (Gomes et al., 2005) emplea un algoritmo que calcula el flujo de carga y toma decisiones de reconfiguración minimizando pérdidas eléctricas. Desde el punto de vista de la confiabilidad vemos a (Popovic y Popovic, 2004) que proponen un algoritmo jerárquico multiobjetivo que permite emplear múltiples funcionales que consideran pérdidas, confiabilidad y energía no suministrada, o bien (Rodriguez y Vargas, 2005) que han propuesto un método heurístico que usa lógica difusa y minimiza el tiempo de restauración del servicio.

En este trabajo se formula y se presenta un algoritmo basado en la Programación Dinámica, que permite el ajuste de la red de MT para minimizar un funcional de costo que contempla indicadores de Calidad de Potencia, Costos de Operación y de Generación en presencia de Generación Distribuida, por parte de los usuarios o de la ED, utilizando ponderaciones individuales para cada aspecto que integra el funcional. El desempeño del algoritmo se evaluó utilizando un sistema de pruebas sencillo y luego se procedió a evaluarlo en un sistema real.

\section{INDICADORES QUE SE ANALIZAN EN RÉGIMEN EQUILIBRADO CONSIDERANDO FALLAS}

De la red en estudio, se tomaron los resultados publicados (Piumetto y Gómez Targarona, 2011) del estado de régimen estacionario equilibrado, en la cual las fuentes de GD son simuladas como barras de generación a potencia activa constante, con valor de despacho fijo en función de la carga máxima del usuariogenerador. Las simulaciones se realizaron con diferentes escenarios de carga, ordenando los resultados para los tres puntos que se analizan, Barra Distribuidora, TOLEDO y TOL II de la red de la Fig. 2, y se definen para este régimen como variables de estado a: a) Los perfiles de tensión en p.u. de cada punto analizado de acuerdo a Ec. (1), b) Las Pérdidas de Potencia Activa en la red en estudio respecto a la configuración radial s/GD de acuerdo a Ec. (2), y c) La carga porcentual de la línea de Alta Tensión como se expresa en Ec. (3). De acuerdo a los resultados obtenidos del estudio (Piumetto y Gómez Targarona, 2011) se logran aspectos técnicos positivos en el régimen estacionario con GD, tanto para el usuario como para la ED, en cuanto a Calidad de Potencia, confiabilidad, seguridad y continuidad de suministro eléctrico. Los perfiles de tensión mejoran y se equiparan; en cuanto a las pérdidas activas el estudio de Energía indica que es variable para cada caso y se mejora el aprovechamiento de las capacidades de las líneas. Ante 
fallas, las respuestas de los valores de las Corrientes de Cortocircuito (ICC) no alcanzan montos importantes, las sobretensiones transitorias se encuentran dentro de los límites fijados por las normas mientras que las relaciones de las impedancias, muestran valores característicos de este tipo de red.

Vp.u. $=\frac{V_{f}}{V_{f N}}$

Dónde: $\quad V_{f}$ Tensión de Fase y $V_{f N}$ Tensión de Fase Nominal

$W p a=\sum W o T+\sum W c u T+\sum W c u L$

Dónde:

$W p a$ es la potencia activa de pérdida total del sistema en estudio

$\sum W o T$ es la potencia de pérdida en vacío de los transformadores de 10 MVA y de los de la GD

$\sum W c u T$ es la potencia de pérdida en el cobre de los transformadores de 10 MVA y de los de la GD

$\sum W c u L$ es la potencia de pérdida en el cobre en las líneas de Media Tensión de la red

$F C L A T=\frac{I C}{I N}$

Dónde: $I_{C}$ Corriente de Carga y $I_{N}$ Corriente Nominal

Se tomaron los resultados del estudio (Piumetto y Gómez Targarona, 2013) sobre las simulaciones de falla monofásica (representan entre el 50 y $70 \%$ de las fallas del sistema y que afectan a más de una fase de la red ya que se considerarán un único evento) y trifásica, en cada punto elegido para el estudio del sistema. Durante el régimen estacionario equilibrado se consideró que las fallas monofásicas y trifásicas, que se producen en el inicio de cada alimentador (D3 o D4); y se definen como variables de estado a los índices de: a) Tensión previa de falla definida en Ec. (4) (siendo esta tensión previa a la falla, uno de los factores determinantes de la capacidad de los equipos sensibles, ya que representa la energía almacenada y disponible para soportar el hueco de tensión, que permite determinar la inmunidad cuando la tensión previa a la falla es menor al 100\%), b) Hueco de tensión en la fase fallada (que por definición en las normas, el hueco de tensión es la disminución del valor eficaz de la tensión de 0,9 p.u. a 0,1 p.u. Norma IEEE o 0,01 p.u. Norma IEC, con duraciones de 0,5 ciclos a un minuto como se define en Ec. (5)), y c) El valor de la corriente de cortocircuito (verificando que los valores de las Icc se han incrementado, siendo las Icc IK3 más significativas que las fallas monofásicas y que tienen igual comportamiento que las monofásicas).

$V a=V b=V c$

Dónde: $V_{a}$ Tensión de Fase

$V h i=\frac{V h}{V N} \times 100$

Dónde: Vh Mínima Tensión del Hueco y VN Tensión Nominal

\section{INDICADORES QUE SE ANALIZAN EN RÉGIMEN DESEQUILIBRADO}

En los sistemas de potencia, el desbalance de tensión y corriente es una ineficiencia importante que no debe subestimarse, dado que las potencias involucradas son superiores a las necesarias en un sistema homólogo simétrico. En general los efectos del desbalance se resumen en la aparición de componentes de secuencia inversa y homopolar que dan como resultado pérdidas adicionales de potencia y energía, calentamiento adicional de máquinas (que limita la capacidad de carga nominal), reducción en el transporte de potencia en los sistemas de distribución y propagación del desbalance a otros nodos de conexión de la red (Piumetto et al., 2014; Gómez Targarona 2005).

De acuerdo a las normativas IEC e IEEE, el factor de desbalance de tensión (FDV) se expresa como Ec. (6), donde V2 corresponde a la componente de secuencia negativa de la tensión y V1 corresponde a la componente de secuencia positiva de la tensión:

$F D V=v_{2} / v_{1} \times 100$

Dónde: $V_{2}$ Tensión de Secuencia Inversa y $V_{1}$ Tensión de Secuencia Positiva 
Tomando la definición del factor de asimetría de KA y de Desclasificación (Piumetto et al, 2014):

$$
\begin{aligned}
& K A=K_{v 2} x K_{i 2}+K_{v 0} x K_{i 0} \\
& D=1-K A
\end{aligned}
$$

Se toman para el estudio los indicadores de Calidad de Potencia (CP) formulados en las Ecs (6) y (8), en los puntos definidos del sistema para considerar las especificaciones de las ED y la de los usuarios.

\section{PROGRAMACIÓN DINÁMICA Y ALGORITMO USADO}

El objetivo de la programación dinámica es resolver un problema de optimización. Se formula un problema de control óptimo con un modelo dinámico del proceso, en éste caso el modelo es tabulado, proponiendo un funcional de costo y se resuelve numéricamente el problema. Se emplea un funcional de costo definido en Ec. (9):

$$
J\left(x_{k}, u_{k}\right)=\sum_{k=1}^{24}\left(x_{k}-x_{D}\right)^{T} \Gamma\left(x_{k}-x_{D}\right)+r \cdot u_{k}^{2}
$$

Donde $\boldsymbol{x}_{\boldsymbol{k}}$ es el vector de estados, $\boldsymbol{x}_{\boldsymbol{D}}$ es el vector de estados deseado y $u_{k}$ la acción de control o decisión tomada. La matriz $\Gamma$ y el escalar $r$ son parámetros de diseño de criterio de optimización. El modelo del proceso, es tabulado y puede representarse como la Ec. (10):

$$
\mathrm{x}_{\mathrm{k}+1}=\mathrm{f}\left(\mathrm{x}_{\mathrm{k}}, \mathrm{u}_{\mathrm{k}}, \mathrm{W}_{\mathrm{k}}\right)
$$

Donde $\mathrm{f}(\cdot)$ es una tabla o base de datos numérica, $\mathrm{x} \in \mathfrak{R}^{6}, u_{k}$ es escalar, y $W_{k}$ es una perturbación medible pero no determinística, $k$ es la variable de etapa que varía entre 1 y 24 que corresponde a las horas del ciclo diario. Dada la formulación del problema, y debido a que no se cuenta con un modelo explícito del proceso, se propone el uso de la Programación Dinámica de Aprendizaje Q (Sutton Barto, 1998) (Chia-Fen y Chun-Ming, 2009) (Chunlin et al., 2013) (Zhou et al., 2011). El objetivo de la programación dinámica es hallar la política de decisiones o ley de control óptima del tipo como se expresa en Ec. (11):

$$
u_{k}=\mu\left(x_{k}, W_{k}\right)
$$

Se busca que minimice al funcional de la Ec. (9) y haga evolucionar al proceso descripto por la representación tabular expresada como en la Ec. (10) desde la etapa 1 hasta la etapa 24. Se implementó el Aprendizaje $Q$ en Matlab, para un problema de $\boldsymbol{x}_{\boldsymbol{i}}$ variables de estado y 1 variable de control. Se asume que la variable u toma 6 valores diferentes, de acuerdo a Tabla 1. La expresión que resume al algoritmo es expresada por la Ec. (12):

$$
\begin{array}{r}
Q_{i, u}(n+1)=\left(1-\gamma_{n}\right) Q_{i, u}(n)+ \\
\gamma_{n} I(x, u)+J^{*}(j)
\end{array}
$$

Donde se asume que el proceso evoluciona del estado $i$ al estado $j$, aplicando la acción de control $u$, lo que genera un costo incremental $I(\cdot)$, en la iteración $n$. La función $\gamma$ depende de la iteración $n$ y se fijó como está expresada en la Ec. (13):

$\gamma_{\mathrm{n}+1}=\frac{100}{100+\eta_{\mathrm{i}, \mathrm{u}}}$

Donde la variable $\eta_{i, u}$ es un contador de veces en la que se visita un determinado estado-acción. Al J*(.) se lo calcula en cada iteración mediante la expresión (14):

$\mathrm{J}^{*}(\mathrm{i})=\min _{\mathrm{u}} \mathrm{Q}_{\mathrm{i}, \mathrm{u}}(\mathrm{n}) \quad \forall \mathrm{i}$. 
El primer paso es calcular a la expresión (14) para una política aleatoria, y luego se mejora mediante el paso de actualización de los factores Qi,u, mediante la Ec. (12). Se actualizan además los contadores de cada uno de los estado-acción, mediante la Ec. (13) y se procede a hallar los nuevos valores de $\mathrm{J}^{*}($.) mediante la Ec. (14). En éste caso se fijaron 50 iteraciones del algoritmo y se observó la convergencia del sistema cuyo esquema se observa en la Fig. 1:

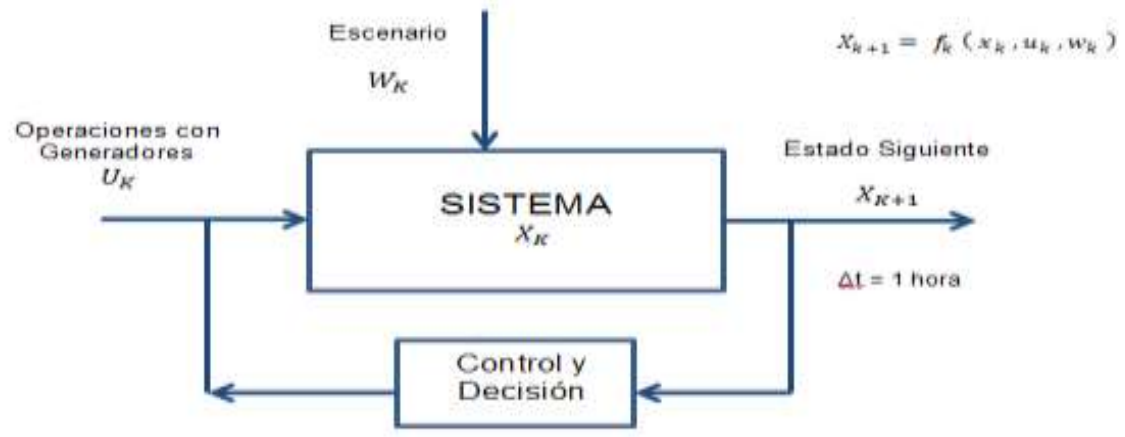

Fig. 1: Esquema del Sistema y su control

\section{RED ESTUDIADA Y DEFINICIÓN DE VARIABLES DE ESTADO}

El sistema eléctrico en estudio abastece la zona de Río II y Pilar de la Provincia de Córdoba, Argentina, corresponde a la empresa EPEC (Empresa Provincial de Energía de Córdoba), y posee demandas agroindustriales, comerciales y residenciales como se aprecia en la Fig. 2. El sistema seleccionado comprende una línea de $66 \mathrm{kV}$, una barra de 13,2 kV en Río II y dos alimentadores de 13,2 kV, denominados D3 y D4 como se observa en diagrama unifilar (Piumetto y Gomez Targarona, 2013). En el esquema radial se indican los valores de carga máxima registrados durante el año 2009 para cada subestación transformadora, y además se muestran los clientes singulares, industrias y resto de las cargas.

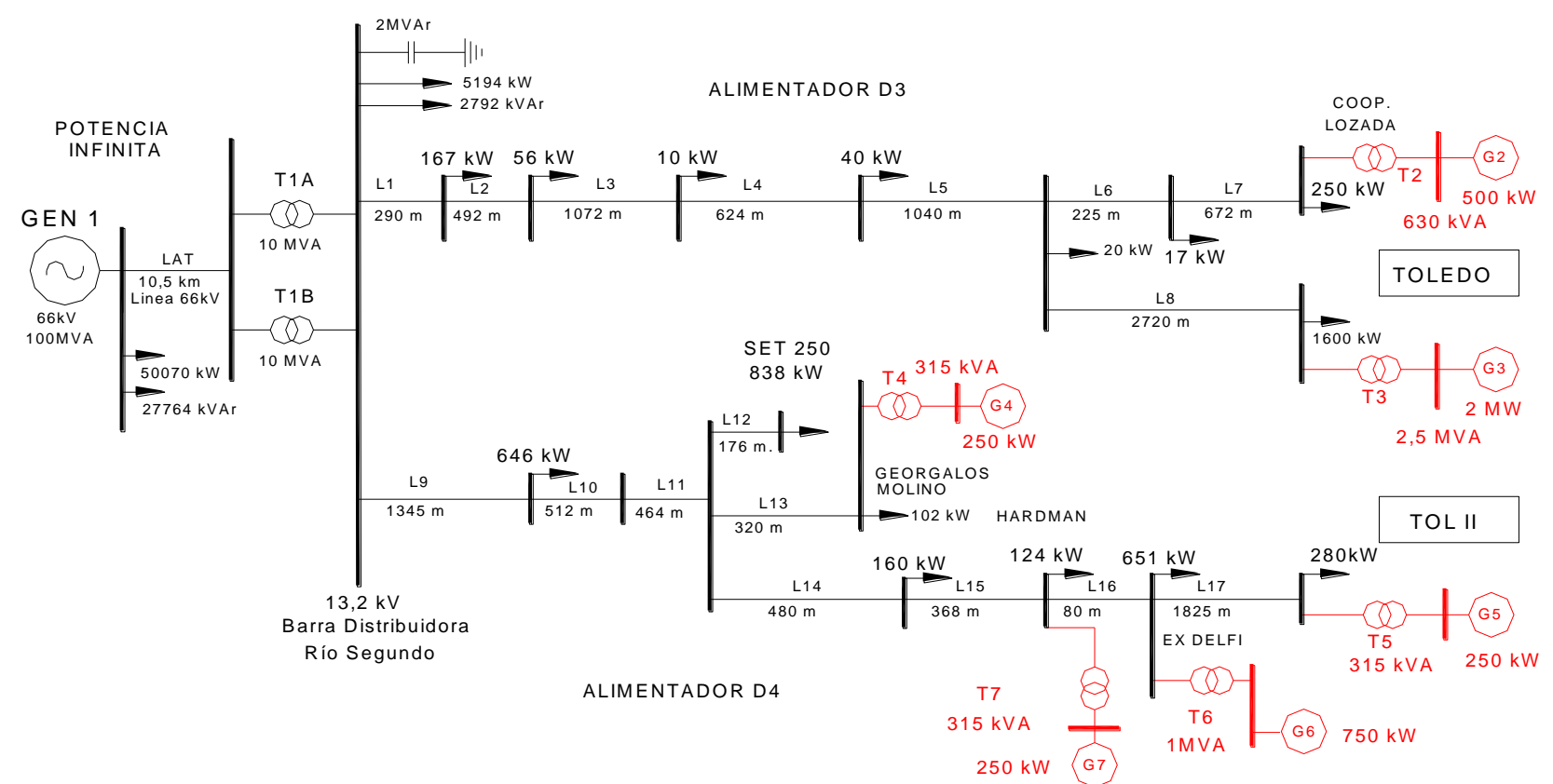

Fig. 2: Diagrama Unifilar del Sistema Eléctrico en estudio, con indicación de la carga máxima al 100\% en los alimentadores y la posición de los GD

Se fijó el criterio de que la GD inyectará valores de potencia activa constantes como: 1/4 de la carga máxima, igual valor de la carga máxima, 3 veces la carga máxima, potencia activa igual a la carga en el resto de los clientes singulares y finalmente con todos los generadores simultáneos. En todos los casos se analizan las configuraciones Radial sin GD y Radial con GD. Se plantearon las siguientes situaciones del estado de carga del sistema: con el $25 \%, 50 \%, 75 \%, 100 \%$ y $125 \%$ del valor de carga máxima registrada no simultánea. Se realiza el estudio de simulación con el software ATP/EMTP para una ventana de $500 \mathrm{~ms}$. Se 
ubicaron dispositivos de medición de potencia, energía, tensión y corriente para el registro de valores instantáneos y máximos en tres puntos del sistema, procesando las magnitudes fasoriales a componentes simétricas y de los indicadores, mediante el uso del software MATLAB.

El registro y análisis se centró en la toma de datos, procesamiento y clasificación de las magnitudes eléctricas en tres puntos en el sistema de MT. Dichos puntos son: a) La barra distribuidora de 13,2 kV de Rio II de la ED, b) En el usuario de la Cooperativa de Toledo al final del alimentador D3, marcado como "TOLEDO", en el cual se han tomado registros en el nivel de MT como en el de BT, y c) El punto del usuario de la Cooperativa de Toledo en el alimentador D4, marcado como "TOL II", en el cual se han tomado registros en el nivel de MT y BT. Luego de procesar todas las mediciones en diferentes situaciones, se procedió a confeccionar una tabla con todas las mediciones del estado de la red para cada situación de carga, estado equilibrado o desequilibrado, o en falla e inyección de la GD. La caracterización del sistema eléctrico utilizando programación dinámica, se realiza tomando los índices de calidad descriptos para cada punto medido del sistema como variables de estado, considerando sus valores óptimos a alcanzar los fijados por las normativas de Calidad de Potencia. Por ello, la decisión que se puede tomar en cada etapa será la cantidad de Generación Distribuida a emplear o tomar, mientras que las perturbaciones del entorno están fijadas por la demanda eléctrica y la ocurrencia de fallas aleatorias en los alimentadores. La curva de carga diaria (24 horas) se modela con escalones que abarcan las distintas situaciones de carga descriptas, como se define en la normativa IEC 60354. Se muestran en las Tablas 1 y 2 las Variables de Control y la Curva de Carga Diaria usada. En las Tablas 3 y 4 se definen las Variables de Estado tomadas en los Regímenes Estacionario Desbalanceado y Balanceado respectivamente:

Tabla 1: Variables de Control

\begin{tabular}{|c|c|c|c|c|}
\hline $\begin{array}{c}\text { Variables } \\
\text { de Control }\end{array}$ & $\begin{array}{c}\text { Potencia } \\
\text { inyect. de } \\
\text { GD en kW }\end{array}$ & $\begin{array}{c}\text { Costo } \\
\text { de Gen. } \\
\text { y Oper. }\end{array}$ & $\begin{array}{c}\text { Punto del } \\
\text { Sistema } \\
\text { Eléctrico }\end{array}$ & $\begin{array}{c}\text { Cantidad } \\
\text { de } \\
\text { Generad }\end{array}$ \\
\hline U0 & 0 & 0 & & 0 \\
\hline U1 & 463 & 1 & $\begin{array}{c}\text { TOLEDO Y } \\
\text { TOL II }\end{array}$ & 2 \\
\hline U2 & 1750 & 2 & $\begin{array}{c}\text { Clientes } \\
\text { Singulares }\end{array}$ & 4 \\
\hline U3 & 1850 & 3 & $\begin{array}{c}\text { TOLEDO Y } \\
\text { TOL II }\end{array}$ & 2 \\
\hline U4 & 6150 & 4 & $\begin{array}{c}\text { TOLEDO Y } \\
\text { TOL II }\end{array}$ & 2 \\
\hline U5 & 7900 & 5 & $\begin{array}{c}\text { Todos los } \\
\text { Generad }\end{array}$ & 6 \\
\hline
\end{tabular}

Tabla 3: Variables de Estado en Régimen Desbalanceado

\begin{tabular}{|c|c|c|c|c|}
\hline $\begin{array}{c}\text { Variables } \\
\text { de Estado } \\
\text { Xi }\end{array}$ & \multicolumn{2}{|c|}{\begin{tabular}{|c|}
$|2|$ \\
Calidad
\end{tabular}} & $\begin{array}{c}\text { Punto del } \\
\text { Sistema Eléctrico }\end{array}$ & $\begin{array}{c}\text { Valor } \\
\text { Óptimo }\end{array}$ \\
\hline X1 & KV2 & FDV & $\begin{array}{c}\text { Barra } \\
\text { Distribuidora ED }\end{array}$ & 0 \\
\hline X2 & D & $\begin{array}{c}\text { Desclasi- } \\
\text { ficación }\end{array}$ & $\begin{array}{c}\text { Barra } \\
\text { Distribuidora ED }\end{array}$ & 1 \\
\hline X3 & KV2 & FDV & Usuario TOLEDO & 0 \\
\hline X4 & D & $\begin{array}{c}\text { Desclasi- } \\
\text { ficación }\end{array}$ & Usuario TOLEDO & 1 \\
\hline X5 & KV2 & FDV & Usuario TOL II & 0 \\
\hline X6 & D & $\begin{array}{c}\text { Desclasi- } \\
\text { ficación }\end{array}$ & Usuario TOL II & 1 \\
\hline
\end{tabular}

Tabla 2: Curva de Carga Diaria de acuerdo a criterio de la IEC 354

\begin{tabular}{|c|c|c|c|c|}
\hline \multirow[b]{2}{*}{$\begin{array}{l}\text { Modelización de } \\
\text { la Demanda }\end{array}$} & & \multirow[b]{2}{*}{$\begin{array}{c}\text { Carga del } \\
\text { Sistema en } \\
\text { referencia } \\
\text { Carga Máxima }\end{array}$} & \multirow{3}{*}{$\begin{array}{l}\text { Ciclo } \\
\text { Diario } \\
\text { total hs }\end{array}$} \\
\hline & Hora & $\begin{array}{l}5 \text { del } \\
\text { a }\end{array}$ & & \\
\hline W25 & 0 & 7 & al $25 \%$ & \\
\hline W50 & 7 & 12 & al $50 \%$ & \multirow{5}{*}{24} \\
\hline W75 & 12 & 17 & al $75 \%$ & \\
\hline W100 & 17 & 20 & al $100 \%$ & \\
\hline W125 & 20 & 23 & al $125 \%$ & \\
\hline W25 & 23 & 24 & al $25 \%$ & \\
\hline
\end{tabular}

Tabla 4: Variables de Estado en Régimen Estacionario Equilibrado

\begin{tabular}{|c|c|c|c|c|}
\hline $\begin{array}{c}\text { Variables } \\
\text { de Estado } \\
\text { Xi }\end{array}$ & \multicolumn{1}{|c|}{$\begin{array}{c}\text { Indicador de } \\
\text { Calidad }\end{array}$} & $\begin{array}{c}\text { Punto del } \\
\text { Sistema } \\
\text { Eléctrico }\end{array}$ & $\begin{array}{c}\text { Valor } \\
\text { Óptimo }\end{array}$ \\
\hline X1 & $\mathrm{V}$ & $\begin{array}{c}\text { Nivel de } \\
\text { Tensión }\end{array}$ & $\begin{array}{c}\text { Barra } \\
\text { Distribuidor } \\
\text { a ED }\end{array}$ & 1 \\
\hline X2 & $\mathrm{V}$ & $\begin{array}{c}\text { Nivel de } \\
\text { Tensión }\end{array}$ & $\begin{array}{c}\text { Usuario } \\
\text { TOLEDO }\end{array}$ & 1 \\
\hline X3 & $\mathrm{V}$ & $\begin{array}{c}\text { Nivel de } \\
\text { Tensión }\end{array}$ & $\begin{array}{c}\text { Usuario } \\
\text { TOL II }\end{array}$ & 1 \\
\hline X4 & A & $\begin{array}{c}\text { Capacidad } \\
\text { Línea }\end{array}$ & Línea AT & 0 \\
\hline X5 & W & $\begin{array}{c}\text { Pérdidas } \\
\text { Activas }\end{array}$ & Del Sistema & 0 \\
\hline
\end{tabular}


En la Tabla 5 se muestran las Variables de Estado definidas y tomadas para el Funcional de Costo de la Ec. (9) considerando fallas monofásicas y trifásicas, en todos los estados se plantea el siguiente conjunto de restricciones basado en la normativa técnica vigente de calidad:

$$
\begin{aligned}
& V_{i \min } \leq V_{x i} \leq V_{\text {imax }} \\
& V_{h \min } \leq V h i \leq V_{h \max } \\
& K_{v 2} \leq K_{v 2 \max } \\
& D \cong 1
\end{aligned}
$$

\begin{tabular}{|c|c|c|c|c|c|c|}
\hline Punto de Medición & Definiciones & $\begin{array}{l}\text { Vector de } \\
\text { Variable de } \\
\text { Estado }\end{array}$ & Unidad & $\begin{array}{l}\text { Valor } \\
\text { Mínimo }\end{array}$ & $\begin{array}{l}\text { Valor } \\
\text { Optimo }\end{array}$ & $\begin{array}{l}\text { Valor } \\
\text { Máximo }\end{array}$ \\
\hline Barra Distribuidora & \multirow{3}{*}{ Tensiones Nominales } & $\mathrm{X} 1$ & $\mathrm{pu}$ & 0,92 & 1 & 1,08 \\
\hline TOLEDO & & $\mathrm{X} 2$ & $\mathrm{pu}$ & 0,92 & 1 & 1,08 \\
\hline TOL II & & X3 & $\mathrm{pu}$ & 0,92 & 1 & 1,08 \\
\hline LAT & Capacidad Línea AT & $\mathrm{X} 4$ & $\mathrm{pu}$ & & 0 & 1 \\
\hline General & Pérdidas Activas & $\mathrm{X} 5$ & W & & 0 & \\
\hline Barra Distribuidora & Huecos de Tensión & $\mathrm{x} 6$ & $\mathrm{pu}$ & 0,1 & 0,9 & \\
\hline TOL II & Huecos de Tensión & $\mathrm{X} 7$ & $\mathrm{pu}$ & 0,1 & 0,9 & \\
\hline en Relé D3R & Corrientes de CC & $\mathrm{x} 8$ & $\mathrm{pu}$ & & 0 & 1 \\
\hline Barra Distribuidora & Huecos de Tensión & $\mathrm{X} 9$ & $\mathrm{pu}$ & 0,1 & 0,9 & \\
\hline TOL II & Huecos de Tensión & $\mathrm{X} 10$ & $\mathrm{pu}$ & 0,1 & 0,9 & \\
\hline en Relé D3R & Corrientes de CC & $\mathrm{X} 11$ & $\mathrm{pu}$ & & 0 & 1 \\
\hline Barra Distribuidora & Huecos de Tensión & $\mathrm{X} 12$ & $\mathrm{pu}$ & 0,1 & 0,9 & \\
\hline TOLEDO & Huecos de Tensión & $\mathrm{X} 13$ & $\mathrm{pu}$ & 0,1 & 0,9 & \\
\hline en Relé D4R & Corrientes de CC & $\mathrm{X} 14$ & $\mathrm{pu}$ & & 0 & 1 \\
\hline Barra Distribuidora & Huecos de Tensión & $\mathrm{X} 15$ & $\mathrm{pu}$ & 0,1 & 0,9 & \\
\hline TOLEDO & Huecos de Tensión & $\mathrm{X} 16$ & $\mathrm{pu}$ & 0,1 & 0,9 & \\
\hline en Relé D4R & Corrientes de CC & $\mathrm{X} 17$ & $\mathrm{pu}$ & & 0 & 1 \\
\hline
\end{tabular}

Tabla 5: Variables de Estado en Régimen Equilibrado considerando Restricciones y Fallas

\section{RESULTADOS Y ANÁLISIS}

Se analizan distintas combinaciones para la ED en cada estado y situación de la red, en la cual se pondera en un primer análisis solamente a la CP, mientras que los Costos de Generación no poseen incidencia. Luego se analiza tomando sus pesos de manera repartida por igual para la CP como para los Costos de Generación. Por último se analiza la incidencia solamente de los Costos de Generación y no de la Calidad de Potencia, observando que los puntos óptimos para la planificación de la red por parte de la ED cambiarán de acuerdo a cada escenario.

\section{Situación I: Régimen Estacionario Equilibrado:}

En este estado se plantean diferentes ponderaciones para la Calidad de Potencia y para los Costos de Generación, observando en la Fig. 3 la variación de la variable de estado X1 que representa a la $\mathrm{V} \%$ en la Barra Distribuidora de la ED junto a la curva de demanda diaria de acuerdo a la política óptima, luego el gráfico de la Acción de Control u que muestra la opción de generación más conveniente para la ponderación seleccionada, en los restantes gráficos se muestran la evolución del Costo para la Política Óptima y por último el desempeño de la política adoptada como más favorable. En esta situación de régimen estacionario en la que se considera solamente a la CP como objetivo a minimizar, se resalta que la política óptima está basada en la inyección de diferentes niveles de GD durante el ciclo diario, pero siempre con la presencia de la GD se mejora y optimiza el comportamiento de la red. Otro aspecto importante es que la GD ha llevado a la red a trabajar dentro de los límites establecidos por sus restricciones, situación que no cumplía en el caso normal a máxima carga sin GD. 
En la Fig. 4 y 5 se muestra (la ponderación con igual peso a la CP y a los Costos de Generación para el objetivo a minimizar) la variación de la variable de estado $X 1$ que representa a la $V \%$ en la Barra Distribuidora de la ED junto a la curva de demanda diaria de acuerdo a la política óptima conjuntamente con el gráfico de la Acción de Control u que muestra la opción de generación más conveniente para la ponderación seleccionada y en los restantes gráficos se muestran la evolución del Costo para la Política Óptima, y por último el desempeño de la política adoptada como más favorable. Se resalta que la política óptima está basada en la inyección de dos niveles de GD durante el ciclo diario (en el caso anterior fueron tres niveles de inyección de la GD), pero siempre con la presencia de la GD se mejora y optimiza el comportamiento de la red.
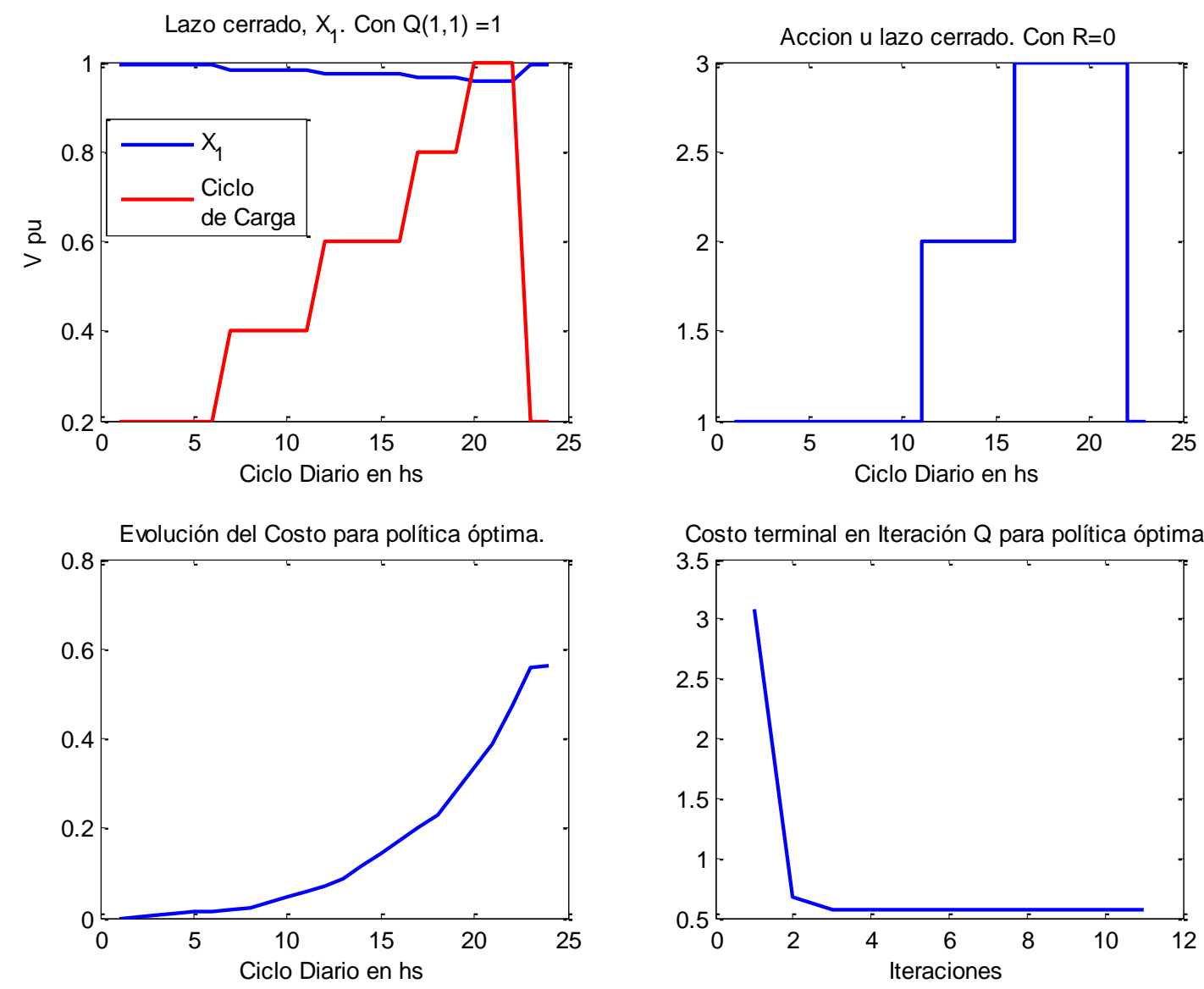

Costo terminal en Iteración $Q$ para política óptima.

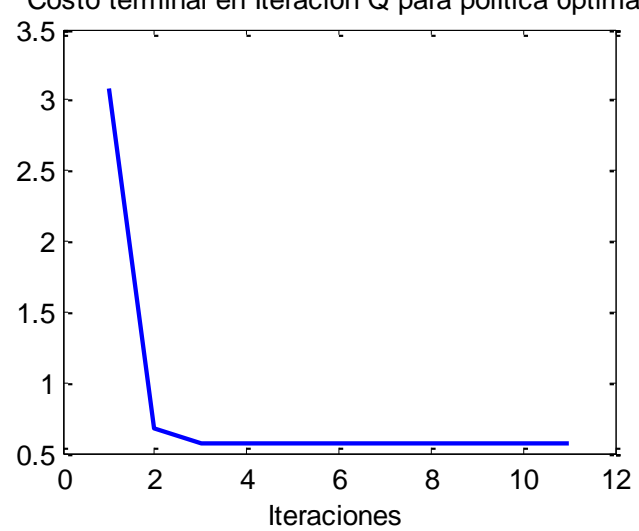

Fig. 3: Evolución de la variable $\mathrm{X}_{1}\left(\mathrm{~V} \% \mathrm{1}_{1}\right)$, de la Acción $\mathrm{u}$, del Costo y las iteraciones para $\mathrm{Q}=1$ (CP) y $\mathrm{R}=0$ (Costos de Generación)
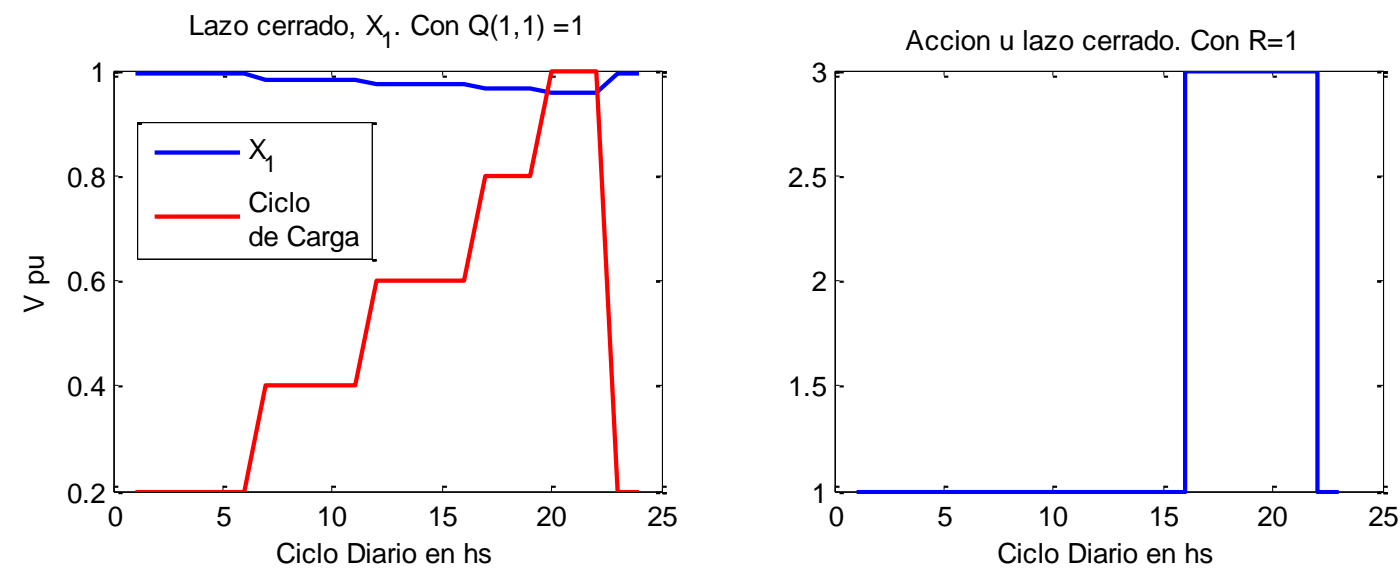

Fig. 4: Evolución de la variable $X_{1}(V \%$ ), de la Acción $u$, para $Q=1(C P)$ y $R=1$ (Costos de Generación) 

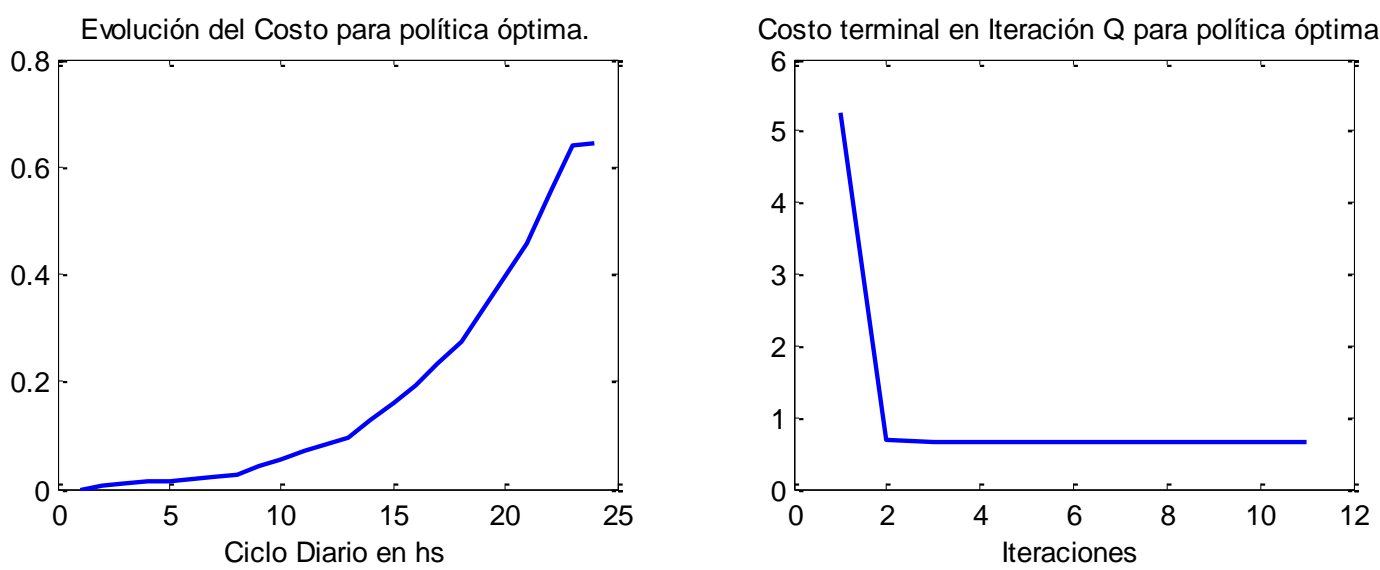

Fig. 5: Evolución del Costo y las iteraciones para $Q=1(C P)$ y $R=1$ (Costos de Generación)

\section{Situación II: Régimen Desequilibrado:}

En este estado se plantean diferentes ponderaciones para la Calidad de Potencia y para los Costos de Generación, observando las gráficas de la variable de estado $X_{1}$ que representa al FDV \% en la Barra Distribuidora de la ED, que se muestra junto a la curva de demanda diaria observando la variación diaria de la variable de estado $X_{1}$ de acuerdo a la política óptima, luego en el gráfico de Acción $u$ se muestra la opción de generación más conveniente para la ponderación seleccionada; en otro gráfico se muestra la evolución del Costo para la Política Óptima y por último con diferentes ponderaciones de la CP y el CG del desempeño de la política adoptada como más favorable, mostrados en las Fig. 6, 7, 8 y 9:
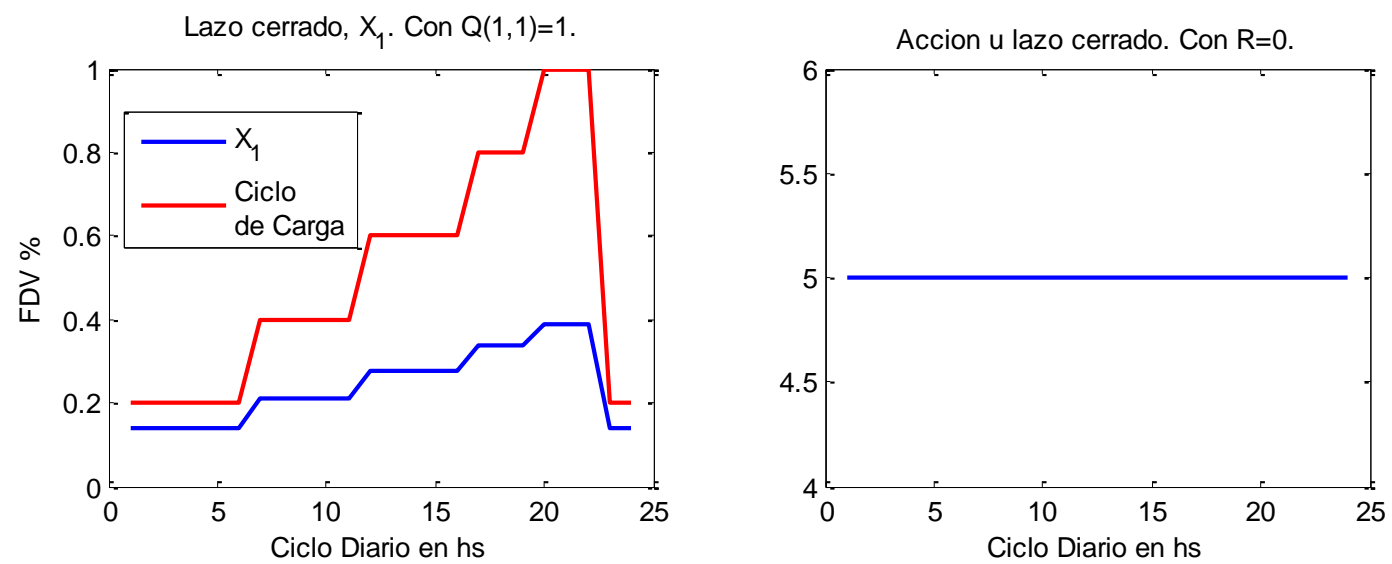

Evolución del Costo para política óptima.

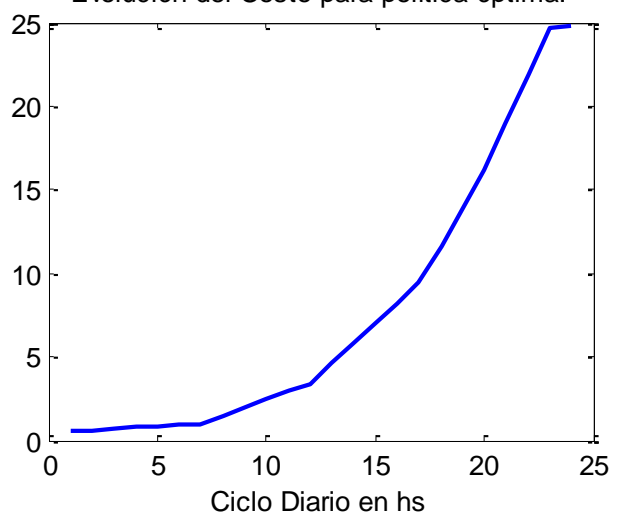

Costo terminal en Iteración $Q$ para política óptima

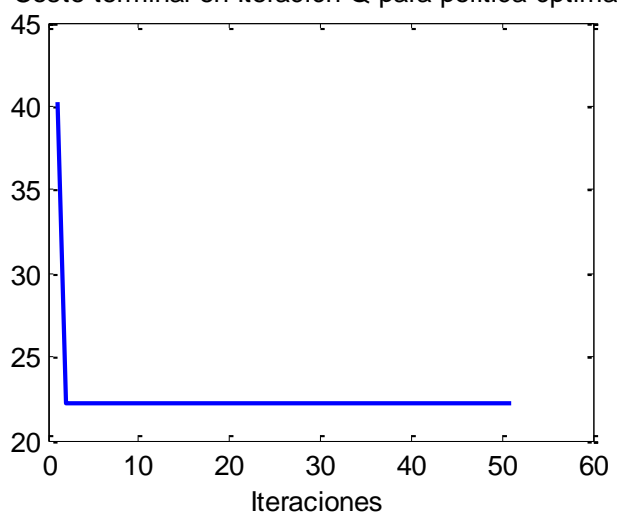

Fig. 6: Evolución de la variable $X_{1}(F V D \%)$, de la Acción $u$, del Costo y las iteraciones para un $\mathrm{Q}=1(\mathrm{CP})$ y $\mathrm{R}=0$ (Costos de Generación) 
Lazo cerrado, $X_{1}$. Con $Q(1,1)=1$.

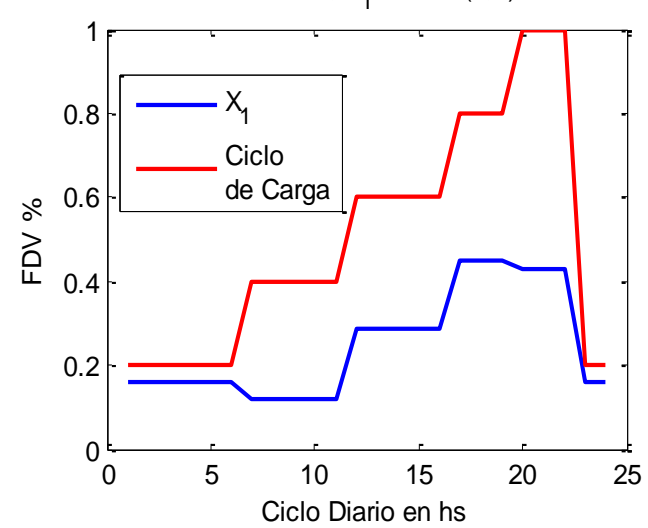

Accion u lazo cerrado. Con $\mathrm{R}=10$.

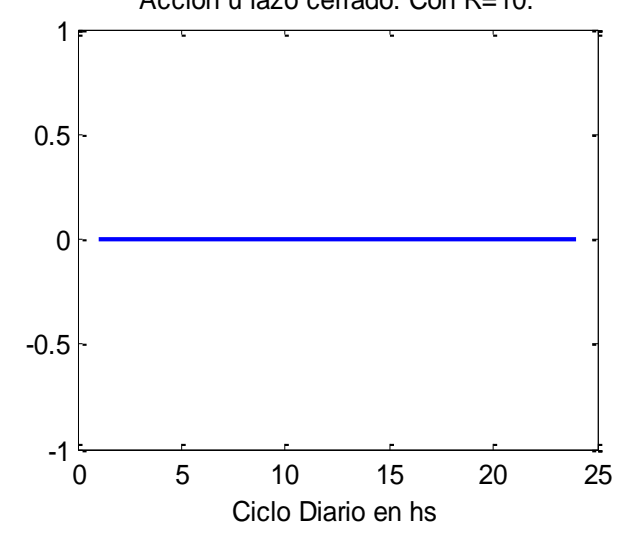

Fig. 7: Evolución de la variable $\mathrm{X}_{1}(\mathrm{FVD} \%)$ y de la Acción de Control para un $\mathrm{Q}=1$ (CP) y $\mathrm{R}=10$ (Costos de Generación)
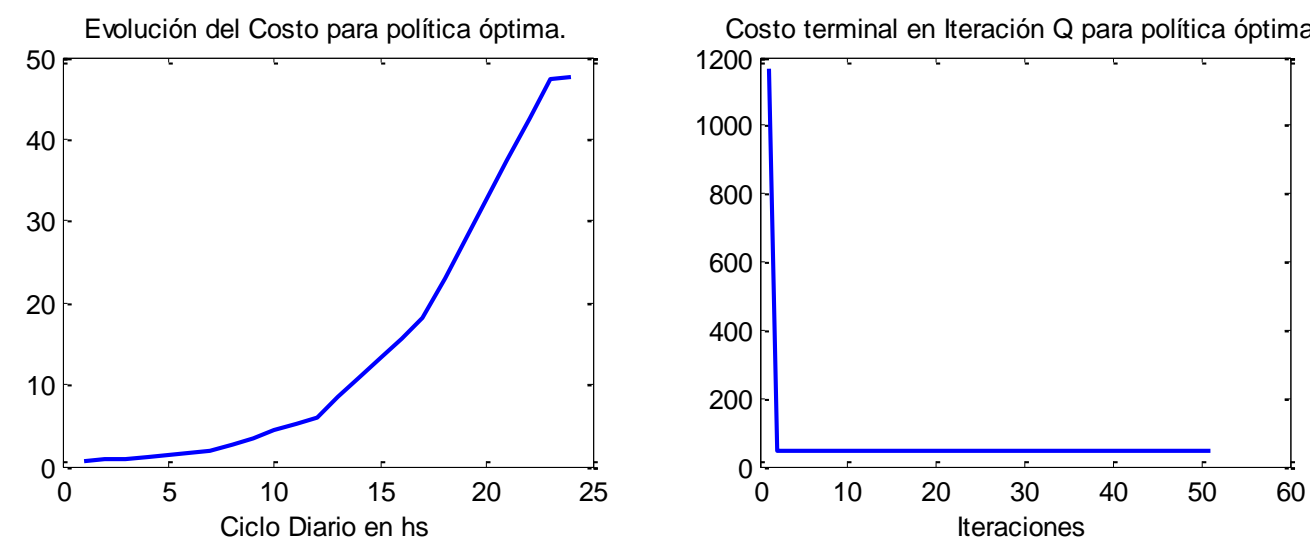

Fig. 8: Evolución del Costo y las iteraciones para un $Q=1(C P)$ y $R=10$ (Costos de Generación)
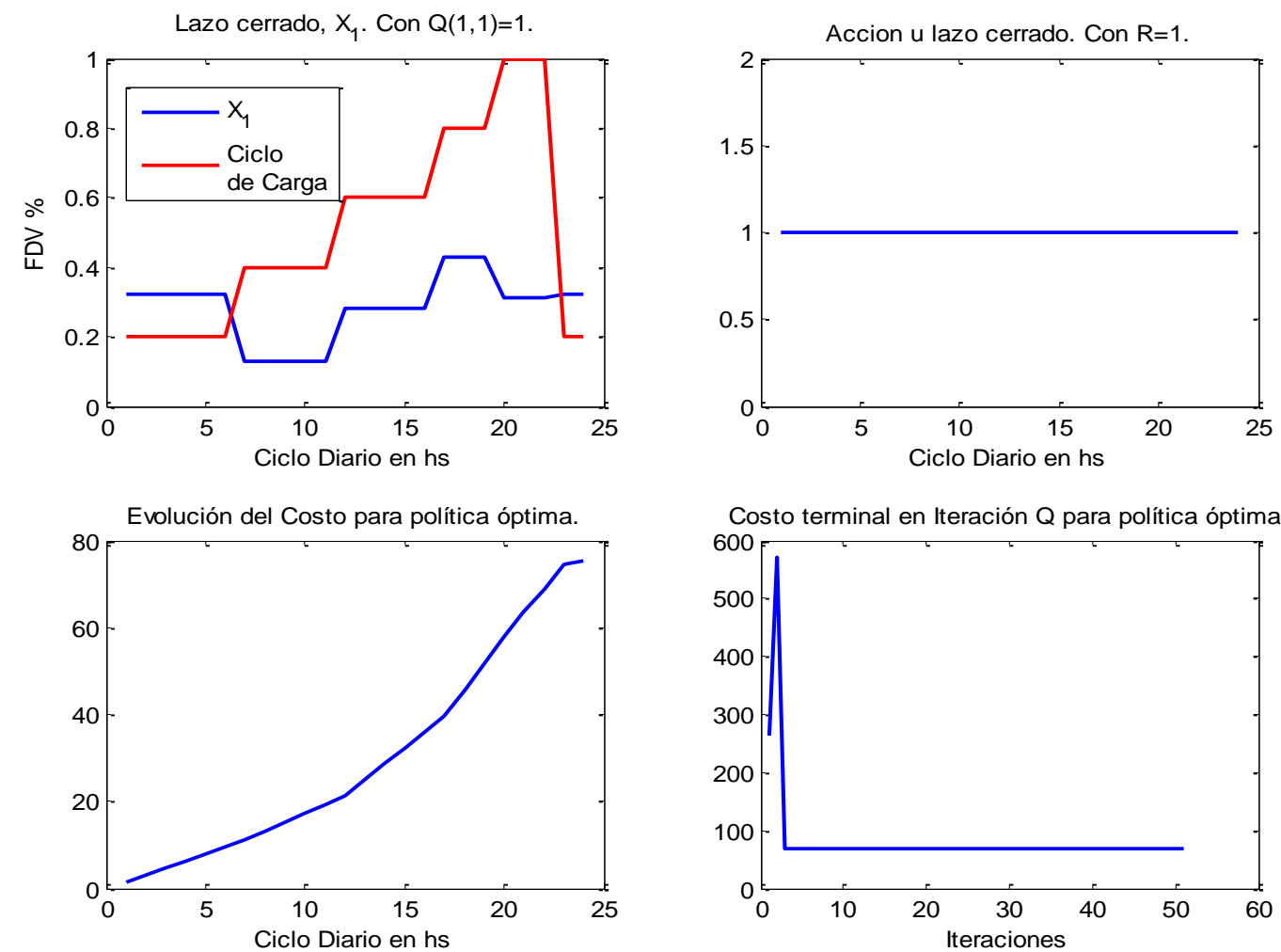

Fig. 9: Evolución de la variable $X_{1}(F V D \%)$, de la Acción $u$, del Costo y las iteraciones para un $\mathrm{Q}=1(\mathrm{CP})$ y $\mathrm{R}=1$ (Costos de Generación) 
En las diferentes corridas del algoritmo $Q$ tanto para situaciones de Régimen Estacionario, Régimen Desequilibrado o con la presencia de falla; si el objetivo es la CP lo que implica ponderar solamente los indicadores de Calidad de Potencia, siempre resulta una política óptima de inyección de GD con diferentes niveles, durante el ciclo diario. Si, por el contrario, el Costo de Generación incide tanto sea por costo directo o por falta de políticas fiscales, este concepto toma relevancia llevando a minimizar la GD o bien prescindir de ella. Si se considera la ponderación por partes iguales a la CP como a los Costos de Generación, aparece siempre con diferentes niveles la inyección de GD en la política óptima. Otro de los aspectos importantes de este estudio, es que la política óptima obtenida lleva al sistema siempre dentro de los límites de operación y de las restricciones técnicas, aun considerando fallas.

Se puede apreciar en las diferentes pruebas, como el algoritmo opera de manera adecuada, y esto se ratifica al observar cómo se minimizan simultáneamente los Costos de Generación, Operación, Pérdidas, y los Indicadores de Calidad de Potencia esperados. La metodología propuesta, determina un conjunto de soluciones viables de balance con diferentes niveles de GD, a considerar según las situaciones que se le plantean a la ED, lo que permite tomar una decisión más acertada por parte del operador de la red cuya operatoria se ejemplifica en la Fig. 10. Nótese que el algoritmo utiliza conjuntos de mediciones del sistema, y no su modelo analítico. La escalabilidad es importante, ya que fácilmente puede sumar mayor número de variables de estado y acciones de control posibles.

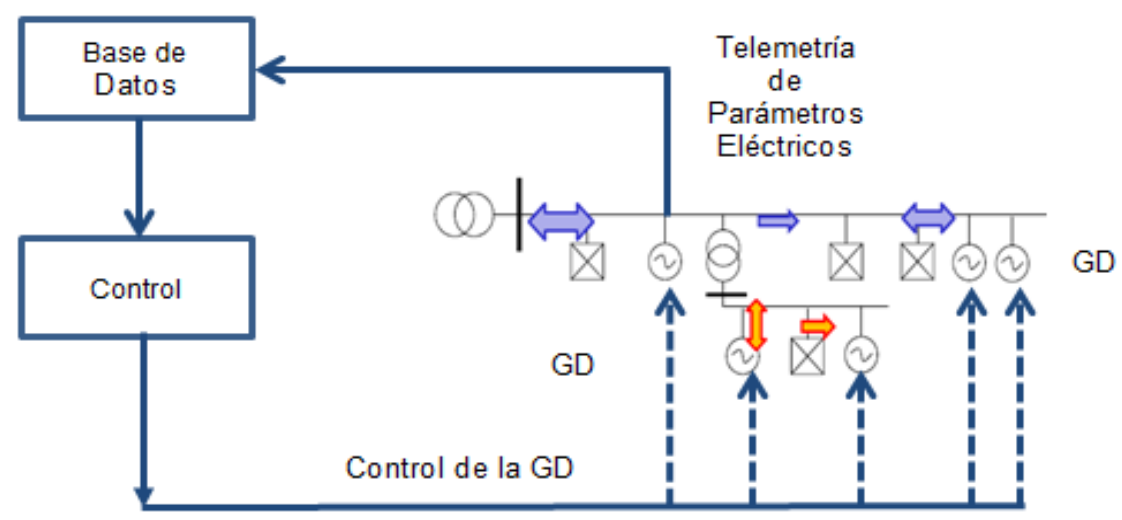

Fig. 10: Esquema de Control para el Sistema Eléctrico de Distribución utilizando Programación Dinámica

\section{CONCLUSIONES}

En el nuevo escenario actual, ingresan al mercado eléctrico nuevos agentes, los cuales son independientes de la ED, con un cierto nivel de acceso a la red de distribución, ofertando inyección de energía al sistema. Ante esta situación, es necesaria la evaluación de decisiones de adquisición de energía, con el fin de cumplir con las normativas, la calidad de potencia y objetivos que rigen este mercado.

Cabe destacar, que los resultados tomados, de los valores entregados por el algoritmo muestran su gran utilidad para el Planificador del Sistema de Distribución, gracias a que, además de proceder adecuadamente con la minimización de las funciones definidas, el mismo explora el espacio de solución y provee variedad de soluciones, con ajuste aceptable dentro de las posibles rutas viables y tipos constructivos.

\section{REFERENCIAS}

Bernal-Agustin J. L., Application of Genetic Algorithms to the Optimal Design of Power Distribution Systems. Ph.D dissertation, Dept. Elect. Eng., Universidad Zaragoza, Zaragosa, España. (1998)

Chia-Feng J. y Chun-Ming L., Ant Colony Optimization incorporated with Fuzzy Q-Learning for Reinforcemente Fuzzy Control. IEEE Transactions on Systems and Cybernetics - Part A: Systemes and Humans, vol 39, № 3 May 2009. (2009)

Chunlin C.; Daoyi D.; Han-Xions L.; Jian C. y Tzyh-Jong L., Fidelity-Based Probabilistic Q-Learning for Control of Quantum Systems. IEEE Transaction on Neural Networks and Learning Systems, Vol $25 \mathrm{~N}^{\circ} 5$, May 2014. (2014) 
Gomes F.V., Carneiro S., Pereira J.L.R., Vinagre M.P., Garcia P.A.N. y Araujo L.R., A new Heuristic Reconfiguration Algorithm for Large Distribution Systems. IEEE Transactions On Power Systems. Vol 20 N $^{\circ}$ 3, pp 1373-1378. (2005)

Gomez-Targarona J.C. Calidad de Potencia: para Usuarios y Empresas Eléctricas. 1ra Edición Editorial Edigar S.A., Buenos Aires, Argentina. (2005)

McDermott T., I. Drezga y R. Broadwater. A. A Heuristic Nonlinear Constructive Method for Distribution Systems Reconfiguration. IEEE Transaction on Power System. Vol 14 № 2, pp 478-483. (1999)

Piumetto M., Gomez Targarona J.C. y Vaschetti J., Reducción de los Factores de Desbalance en un Sistema de Distribución de M.T. debido a la Inserción de Generación Distribuida. Revista CIT Vol. 25 № 4. (2014)

Piumetto M. y Gomez Targarona J.C., Characterization of Voltage Sags and its Impact on Sensitive Loads in a MV System with Distributed Generation for Single-phase Fault. IEEE Latin American Transactions Vol. 11 № 1. (2013)

Piumetto M. y Gómez Targarona J.C., Los Desafíos de la Generación Distribuida, Revista de la Asociación Electrotécnica Argentina, AEA, pp. 20 - 28, Febrero 2011. (2011)

Popovic D. y Popovic Z., A Risk Management Procedure for Supply Restoration in Distribution Networks. IEEE Transactions On Power System. Vol 19 pp 221-228. (2004)

Rodriguez J.R.A., Vargas A., Fuzzy-heuristic Methodology to Estimate the Load Restoration Time in MV Networks. IEEE Transactions on Power Systems. Vol 20 № 2 pp 1095-1102. (2005)

Sutton, Richard S.; Barto, Andrew G., Reinforcement Learning: An Introduction. MIT Press. ISBN 0-26219398-1. Disponible en línea: http://webdocs.cs.ualberta.ca/ sutton/book/ , mayo 2014. (1998)

Zhou B.; Chan K.W. y Yu T., Q-Learning Approach for Hierarchical AGC Scheme of Interconnected Power Grids. Energy Procedia 12 (2011) 43-52, Publised by Elsevier Ltd. (2011) 